\title{
DE ARAÑAS Y CUCARACHAS \\ DEVENIR-ANIMAL Y LA POTENCIA DE LO ABYECTO \\ UN ACERCAMIENTO A LA PASIÓN SEGÚN G. H. Y ARACNE
}

Andrea Torres Armas ${ }^{1}$

Resumen: Esta ponencia analizará brevemente dos obras literarias: La pasión según $G$. $H$. (1964), de la escritora Clarice Lispector, y «Aracne», contenida en el libro VI de las Metamorfosis de Ovidio ( 8 d. C.). El análisis girará en torno a dos ejes: el devenir-animal (devenir-insecto/araña) y la relación devenir-mujer con lo abyecto y lo monstruoso. Finalmente, se planteará una dimensión política-emancipatoria del devenir menor. Para el análisis del devenir se tomará como punto de partida la noción de fuerzas activas y fuerzas reactivas propuesta por Friedrich Nietzsche; en cuanto a lo abyecto, recurriremos a la definición propuesta por Julia Kristeva en Los poderes de la perversión (1980).

Palabras clave: Devenir-animal; devenir-mujer; literatura; lo abyecto.

«A complete life may be one ending in so full identification with the non-self that there is no self to die»

(Bernard Berenson) $^{2}$

«El horror será responsabilidad mía hasta que se complete la metamorfosis y el horror se transforme en luz»

(Lispector) $^{3}$

«Su literatura no es un viaje a través del pasado, su literatura es la de nuestro porvenir»

(Deleuze y Guatatri) $^{4}$

\section{Dos vertientes}

¿Qué tienen en común dos textos tan distantes en tiempo y espacio como son La pasión según G. H. y Aracne? Pienso, en primera instancia, en la dimensión simbólica de que dos mujeres, G. H., una ceramista, en la novela de Lispector, y Aracne, una tejedora en la obra de Ovidio, devengan-insecto - aunque en términos estrictamente taxonómicos las arañas no pertenecen a este orden - . En cierto sentido, este devenir que se nos presenta implica captar en el código de la animalidad lo abyecto y lo monstruoso que tienen en sí la cucaracha en que deviene G. H. y la araña en que lo hace Aracne.

Julia Kristeva describe, en Poderes de la perversión, a lo abyecto como «aquello que perturba una identidad, un sistema, un orden. Aquello que no respeta los límites, los lugares, las reglas, la complicidad, lo ambiguo, lo mixto» ${ }^{5}$. Abyecto es un arrojado, un excluido, que se separa, que no

\footnotetext{
${ }^{1}$ Escritora y correctora de textos. Actualmente estudia literatura en la Universidad de las Artes-Ecuador. E-mail: andrea.torres@uartes.edu.ec.

${ }^{2}$ Del epígrafe de La pasión según G. H.

${ }^{3}$ Clarice Lispector, La pasión según G. H. (Barcelona: Ediciones Siruela, 2013), 17.

${ }^{4}$ Gilles Deleuze y Felix Guattari, Kafka. Por una literatura menor (México D. F.: Ediciones Era, 1990), 120.

${ }^{5}$ Julia Kristeva, Poderes de la perversión. Ensayo sobre Louis-Ferdinad Céline (México: Siglo XXI, 2006 ), 11
} 
reconoce las reglas del juego, y por lo tanto «erra en vez de reconocerse, de desear, de pertenecer o rechazar» ${ }^{6}$. La categoría de la abyección, además de ser parte constituyente del sujeto, se sublima en la dimensión de los discursos culturales y estéticos: el arte, la literatura y la filosofía.

Empecemos resumiendo brevemente las historias:

En La pasión según G. H., una escultora-ceramista, G. H. ${ }^{7}$, ha despedido a la criada. Se decide a limpiar el cuarto que esta ocupaba y se encuentra con dos cosas: un dibujo en la pared y una cucaracha en el clóset. La contemplación de ese insecto, a pesar de la repulsión que le produce, es el desencadenante de una renovación vital. Estos acontecimientos le harán replantearse su existencia y su identidad desde la construcción a partir de fragmentos. En la primera página de la obra la narradora nos sitúa en una búsqueda existencial que oscila entre la ausencia y el despojo, ese es un primer indicio de la búsqueda de una zona de indiferenciación entre lo que se es y lo que no se ha sido:

Si me confirmo y me considero verdadera, estaré perdida, porque no sabría dónde encajar mi nuevo modo de ser; si avanzase en mis visiones fragmentarias, el mundo entero tendría que transformarse para que yo ocupase un lugar en él.

He perdido algo que era esencial para mí, y que ya no lo es. No me es necesario, como si hubiese perdido una tercera pierna que hasta entonces me impedía caminar, pero que hacía de mí un trípode estable. He perdido esa tercera pierna. $\mathrm{Y}$ he vuelto a ser una persona que nunca fui ${ }^{8}$.

Aracne, en cambio, se nos presenta en el ámbito de la mitología. Ovidio nos dice que Aracne fue una gran tejedora que entró en disputa con Palas Atenea (Minerva), diosa de la artesanía y la sabiduría. La diosa, ofendida porque Aracne no se reconoce como su alumna, la reta a un duelo, lo que es únicamente posible porque ambas son vírgenes y, por lo tanto, «aptas para las contiendas de todo tipo» ${ }^{9}$. Este estatuto virginal introduce una primera noción de lo que debes ser/hacer para ser una mujer digna; contar con los atributos para contraefectuar las fuerzas activas, convierte a Aracne en potencia pura. Aracne, que decide plasmar la historia de los amores de los dioses muchas veces transformados en animales para logar la cópula-, sale vencedora en el reto y Minerva la transforma en araña, condenándola a tejer por la eternidad.

Podríamos asegurar que, de cierta manera, estas dos historias se constituyen como novelas de formación (Bildungsroman) — no en términos formales, sino en términos ontológicos— porque se puede apreciar la progresión de la transformación de las protagonistas en el espacio, aunque ciertamente no se desarrolla de manera diacrónica. La metamorfosis no puede ser registrada en el tiempo cronológico, sino que ocurre en el tiempo del Aión; es decir, en la eternidad.

\section{Los devenires:}

- G. H.

François Zouravichvili, explicando a Deleuze, nos dice que:

\footnotetext{
${ }^{6}$ Kristeva, Poderes de la perversión..., 16

7 Nótese que el nombre de la protagonista no se hace explícito y que este hecho puede provocar tantas interpretaciones como lectores. Diremos que G. H. podría ser un guiño a género humano, por lanzar una idea.

${ }^{8}$ Lispector, La pasión según G. H..., 9.

${ }^{9}$ José Carlos Fernández Corte y Josefa Cantó Llorca, en: Pluvio Ovidio Nasón, «Aracne. Libro VI», Metamorfosis

- Libro VI-X (Madrid: Gredos, 2012), 6.
} 
'Devenir', en primer lugar, es sin duda, cambiar: ya no comportarse más ni sentir las cosas de la misma manera; ya no hacer las mismas evaluaciones [...] Devenir significa que los datos más familiares de la vida han cambiado de sentido o que ya no mantenemos las mismas relaciones con los elementos habituales de nuestra existencia [...] Esto quiere decir la inclusión la un afuera: entramos en contacto distinto de nosotros mismos, algo nos pasó. 'Devenir' implica entonces, en segundo lugar, un encuentro: uno se convierte a sí mismo en otro que en relación con otra cosa ${ }^{10}$.

De acuerdo con esto, tanto G. H. como Aracne devienen: G. H. deviene-mujer-sí-misma; y, posteriormente, cucaracha. Aracne, por su parte, devendrá también mujer y luego arácnido.

Los traductores de la obra de Ovidio anotan: «Con su negativa a considerarse alumna de Palas Atenea para asegurar su independencia como artista, Aracne da un paso que ningún poeta de tradición alejandrina se atrevería a dar, a saber, considerarse al margen de toda tradición» ${ }^{11}$. Este, podríamos señalar, es un rasgo de insumisión, un primer gesto emancipatorio.

Con G. H., como se ha dicho, se observan dos momentos clave en su devenir. El primero se da cuando reflexiona sobre su propia transformación, así: «iQué difícil está resultando saber cómo era yo! No obstante, tengo que hacer al menos el esfuerzo de darme una forma primera para poder entender lo que ha sucedido al perder esta forma» ${ }^{12}$. La progresión continúa cuando enuncia: «El resto era el modo en que poco a poco me había transformado en la persona que tiene mi nombre y he terminado por ser mi nombre» ${ }^{13}$. De este instante pasa a la enunciación de la fórmula del devenir (N-1) para acotar cómo ha terminado de devenir-mujer:

Cuando me hallaba sola, no se producía un desfondamiento, tenía solamente un grado menos de lo que yo era ${ }^{14}[. .$.$] Haber hecho escultura durante un tiempo$ indeterminado e intermitente también me daba un pasado y un presente que permitía a los demás situarme [...] y me ha situado, tanto para los demás como para mí misma, en una zona que socialmente se halla entre mujer y hombre. Lo cual me dejaba mucho más libre para ser mujer ${ }^{15}$. Cabe recalcar que el libro de Lispector se escribe catorce años antes que Mil Mesetas y enunciación de la fórmula del devenir. Igual que con Proust o Kafka, la literatura se anticipa al pensamiento de Deleuze y Guattari; sin embargo, es improbable que ellos conocieran la obra de Lispector, publicada en América y en portugués.

El devenir produce también con el encuentro de un dispositivo de identificación consigo misma y con su empleada, Janair, cuya invisibilización la hace abstraerse de sí misma. Este dispositivo es un dibujo en la pared, «la silueta, trazada con carboncillo, de un hombre desnudo, de una mujer desnuda y de un perro que estaba más desnudo que un perro» ${ }^{16}$. El dibujo, dirá la narradora, más que un «adorno, era una escritura» ${ }^{17}$ que le permite ponerse entre comillas y hacer una citación de sí.

\footnotetext{
${ }^{10}$ François Zouravichvili, «QQué es un devenir para Gilles Deleuze?», Reflexiones Marginales. Trad. Verónica Jocelyn Guarneros Rojas. Conferencia pronunciada en Horlieu (Lyon) el 27 de marzo de 1997 (ISSN 155 2007-8501).

${ }^{11}$ Fernández Corte y Cantó Llorca, «Aracne...», 6.

${ }^{12}$ Lispector, La pasión según G. H., 23.

${ }^{13}$ Lispector, La pasión según G. H., 24.

${ }^{14}$ Énfasis propio.

${ }^{15}$ Lispector, La pasión según G. H., 25.

${ }^{16}$ Lispector, La pasión según G. H., 38.

${ }^{17}$ Lispector, La pasión según G. H., 40.
} 
La metamorfosis de G. H. concluye con un devenir cucaracha, es decir, con un devenir animal por medio de la ingesta: «Tenía yo en la boca la materia de una cucaracha, y por fin había realizado el acto ínfimo. // No el acto máximo [...] Por fin mi envoltura se había roto realmente, y yo era ilimitada. Por no ser, yo era ${ }^{18}$.

Recordemos que, como dice Deleuze en Crítica y clínica $^{19}$ : 'Devenir' no es alcanzar una forma, sino encontrar «la zona de vecindad, de indiscernibilidad o indiferenciación»; para que esto ocurra, en el caso de G. H., se debe producir una triple operación: 1) concretización del afuera; 2) la minorización del otro; y, 3) la transformación de fuerzas activas y fuerzas reactivas. El cuerpo de G. H. es el campo de fuerzas donde se producen estas operaciones.

\section{- Aracne}

Había llegado su fama a oídos de la Tritonia (Palas Atenea, diosa del tejido), quien se presenta frente a la meonia Aracne para ser alabada como si la mortal hubiese sido instruida en tal arte por ella. Aracne, de humilde cuna, «lo niega expresamente, y sintiéndose ofendida, aunque su maestra fuera tan grande, dice: "Que se enfrente conmigo; a nada me negaré si me vence" $\rangle^{20}$.

Minerva la confronta y le pide que sea humilde y que le pida perdón a la diosa por su alevosía, pero ella le dice:

Tu entendimiento flaquea, llegas agotada por una larga vejez y te hace daño haber vivido tanto. Que escuchen esos consejos tuyos tus nueras o tus hijas, si es que las tienes. A mí me basta con mi propia sensatez; y para que no creas que has conseguido algo con tus recomendaciones, persisto en la misma resolución ${ }^{21}$.

Aracne, como hemos dicho, busca su independencia como artista-tejedora y eso es una afrenta. Acto seguido, Palas Atenea se revela y empiezan una contienda de tejido sin dilaciones. La diosa exalta al resto del panteón para ratificar su naturaleza divina:

Palas borda la roca de Marte en la acrópolis Cecropia y el vetusto litigio por dar nombre a la comarca. Los doce celícolas, con Júpiter en su centro, se sientan en sus altas sedes con una gravedad imponente; su fisonomía habitual identifica a cada uno de los dioses. La de Júpiter es la imagen de un rey. Al dios del mar lo representa de pie, hiriendo con el largo tridente los ásperos peñascos y haciendo brotar un mar del interior de la herida infligida al peñasco: con esa prenda reivindica la ciudad. A sí misma se da el escudo, se da la lanza de aguzada punta, se da el casco para la cabeza, se defiende el pecho con la égida: también borda cómo la tierra, golpeada por la punta de su lanza, hace brotar un vástago de grisáceo olivo con sus frutos y cómo los dioses admiran el prodigio. Una Victoria pone fin a la obra ${ }^{22}$.

Para Aracne, la relación con su afuera es otra que la de G. H.; está mediada por un el juicio estético al que se someterán los tejidos que son usados como un espacio narrativo desarticulado y autónomo. El tejido es una narración, cuando empieza el duelo, comienza un proceso de escritura, de exteriorización. Deleuze menciona en Crítica y Clínica: «La escritura

\footnotetext{
${ }^{18}$ Lispector, La pasión según G. H., 174.

${ }^{19}$ Gilles Deleuze, Crítica y Clínica (Barcelona: Anagrama, 1996), 12.

${ }^{20}$ Ovidio, «Libro VI, Aracne», Metamorfosis (Madrid: Gredos, 2012), 5.

${ }^{21}$ Ovidio, «Libro VI, Aracne...», 6.

${ }^{22}$ Ovidio, «Libro VI, Aracne...», 6
} 
es inseparable del devenir; escribiendo, se deviene-mujer, se deviene-animal o vegetal, se deviene-molécula hasta devenir-imperceptible» ${ }^{23}$.

La Meónide dibuja a Europa burlada por la apariencia del toro; dirías que el toro y los mares son de verdad. [...] También representó a Asteria, que era sujetada por un águila agresiva, y a Leda, recostada bajo las alas del cisne. Añadió cómo Júpiter, oculto bajo la apariencia de un sátiro, colmó a la hermosa hija de Nicteo con doble prole y cómo fue Anfitrión cuando te conquistó, Tirintia, cómo siendo de oro burló a Dánae y siendo de fuego a la Asópide, siendo pastor a Mnemósine, o pintada serpiente a la Deoide. A ti también te representó, Neptuno, transformado en torvo novillo con la doncella Eólida; tú, en forma de Enipeo, engendras a los Aloídas, como carnero engañas a la Bisáltide. También la suavísima madre de las mieses, de amarilla cabellera, tuvo comercio contigo en forma de caballo; en forma de ave te recibió la madre del caballo alado, cuyos cabellos son culebras, en forma de delfín, Melanto. A todos estos, les prestó la apariencia y el ambiente que les eran propios. Allí está Febo, con aspecto rústico, y la historia de cómo llevó unas veces las alas de un azor, otras la piel de un león y cómo disfrazado de pastor burló a Ise la Macareide; cómo engañó Líber a Erígone bajo la forma de una falsa uva, cómo Saturno, bajo la forma de caballo, creó al bicorpóreo Quirón. La parte extrema de la tela, rodeada de una fina orla, es de flores entretejidas con zarcillos de hiedra ${ }^{24}$.

Al terminar el reto de tejido-escritura, Minerva y Envidia, que son quienes juzgan, no encuentran fallas la narración de Aracne, quien ha plasmado las relaciones de los dioses transfigurados con animales para fecundar a mortales. Ante este acto, la diosa furiosa condena:

Vivirás, insolente Aracne, de esta forma suspendida, tal será tu castigo para toda la posteridad». Al marcharse Minerva, —dice Ovidio- le arrojó el jugo de una hierba envenenada, que le hizo caer los cabellos, la nariz y las orejas; su cabeza y su cuerpo disminuyeron, las piernas y los brazos en patas sutilísimas se tornaron, y el resto del cuerpo no presentó más que un grueso vientre. De esta manera, en araña transformada, sigue tejiendo con sus hilos, tarea a la que estaba acostumbrada ${ }^{25}$.

La metamorfosis de Aracne consiste en volverse innecesaria de la diosa, encarnado en este caso en Palas Atenea. Aracne se encuentra en una necesidad de devenir para contraefectuarla. No es Minerva, la diosa, quien triunfa con esta transformación, sino Aracne, pues logra emanciparse de la mirada del otro. Para su metamorfosis se ha efectuado una doble operación: $1^{\circ}$ ) la concretización del afuera; y, $2^{\circ}$ ) devenir menor.

Esta metamorfosis se configura como una concreción de la voluntad de poder. Aracne, una vez que ha devenido araña lo puede todo, ya que es un conglomerado de fuerzas activas.

Aracne y G. H. han concretado el «acto ínfimo»; es decir que han devenido imperceptibles.

\section{Lo minoritario y la potencia de lo abyecto}

Es importante recordar que para Deleuze 'mayoritario' y 'minoritario', en cuanto al devenir, no remiten a la noción de cantidades que pueden numerarse, sino a categorías

\footnotetext{
${ }^{23}$ Deleuze, Crítica y Clínica..., 5.

${ }^{24}$ Ovidio, «Libro VI, Aracne...», 7.

25 Ovidio, «Libro VI, Aracne...», 13.
} 
relacionadas con el poder ${ }^{26}$. Actualmente, el número de mujeres en el mundo bordea el $52 \%$ de la población y no cabe duda que, desde el punto de vista del poder, somos minoritarias. En Deleuze, sin embargo, la potencia se reafirma justamente en los devenires minoritarios.

Esther Díaz lo explica:

Un devenir no es una imitación de aquello que se deviene. No se devieneanimal pareciéndose a un animal, sino capturándole el código a la animalidad. [...] El bloque de devenir no tiene a otro sujeto que a sí mismo y es involuntario, simplemente acaece ${ }^{27}$.

Las obras que se acaban de analizar cumplen las condiciones del devenir menor, principalmente la involuntariedad y guardan estrechas similitudes. Vemos que en ambas historias el devenir está directamente ligado con la condición existencial, así como con la condición de reproducción material de la escritura (cerámica, dibujo y tejido).

Es importante acentuar el asunto de la involuntariedad y del deseo: por un lado, sabemos que Deleuze se opone al deseo en términos lacanianos; es decir, el deseo como falta; se opone al no-ser, y propone un deseo que sea pura afirmación. La involuntariedad está dada obviamente en las dos protagonistas: ni Aracne se quiere convertir en araña, ni G. H. se quiere convertir en cucaracha; sin embargo, se hallan en esa zona de indiferenciación y captan el código de la animalidad.

La relación de estas dos mujeres convirtiéndose en invertebrados - la cucaracha y la araña, que son animales tradicionalmente considerados indeseables, nos confronta con el tema de lo monstruoso y de lo abyecto: aquello que rechazamos y causa repulsión; sin embargo, el devenir minoritario es un asunto emancipador: G. H. se libera de las ataduras de sí misma y Aracne de la mirada de la diosa. En esta medida, devenir-menor, devenir-mujer, devenir-animal adquiere una dimensión política emancipatoria porque, aunque haya involuntariedad, existe, en ambos casos, el deseo de afirmación de sí mismas. Como diría Julia Kristeva: «La abyección es una resurrección que pasa por la muerte del yo (moi). Es una alquimia que transforma la pulsión de muerte en un arranque de vida, de nueva significancia ${ }^{28}$. Una vez resurrectas estas mujeres, emancipadas, todo lo pueden, pensemos sino en todas las mujeres insurrectas en la mitología: Medusa, Lilith, Lamia, las brujas... Una vez que han sido rechazadas, convertidas en monstruos y, por lo tanto, abyectas, lo pueden todo, no necesitan cumplir con las 'virtudes' y los preconceptos de lo que de ellas se espera; son potencia de sí mismas y pueden escribir su historia, como las arañas, ad infinitum.

\section{Referencias}

DELEUZE, Gilles. Crítica y Clínica. Barcelona: Anagrama, 1996.

DELEUZE, Gilles; GUATTARI, Felix. Kafka. Por una literatura menor. México, D. F.: Ediciones Era, 1990.

. Mil mesetas. Valencia: Editorial Pre-textos, 2002.

\footnotetext{
${ }^{26}$ Gilles Deleuze y Felix Guattari, Mil mesetas (Valencia: Editorial Pre-textos, 2002).

${ }^{27}$ Esther Díaz, «Nietzsche-Deleuze o el devenir animal», en Mónica Cragnolini (comp.), Entre Nietzsche y Derrida: vida, muerte, sobrevida (Buenos Aires: La Cebra, 2013), recuperado de: <https://www.estherdiaz.com.ar/textos/nietzsche deleuze.htm>.

${ }^{28}$ Kristeva, Poderes de la perversión..., 25.
} 
DÍAZ, Esther. Nietzsche-Deleuze o el devenir animal. In: CRAGNOLINI, Mónica (comp.). Entre Nietzsche y Derrida: vida, muerte, sobrevida. Buenos Aires: La Cebra, 2013. $<$ https://www.estherdiaz.com.ar/textos/nietzsche_deleuze.htm $>$.

KRISTEVA, Julia. Poderes de la perversión. Ensayo sobre Louis-Ferdinad Céline. México: Siglo XXI, 2006 [versión pdf].

LISPECTOR, Clarice. La pasión según G. H. Traducción de Alberto Villalba Rodríguez. Barcelona: Ediciones Siruela, 2013.

OVIDIO. Aracne. Libro VI. In: Metamorfosis - Libro VI-X. Traducción y notas de José Carlos Fernández Corte y Josefa Cantó Llorca. Madrid: Gredos, 2012.

ZOURAVICHVILI, François. ¿Qué es un devenir para Gilles Deleuze?. Reflexiones Marginales. Traducción de Verónica Jocelyn Guarneros Rojas. Conferencia pronunciada en Horlieu (Lyon) el 27 de marzo de 1997 (ISSN 155 2007-8501). 